\title{
Uebersicht
}

\section{der von Herrn Carl Euler im District Cantagallo, Provinz Rio de Janeiro, gesammelten Vögel.}

Vom Her a u ge $\mathrm{b} \theta \mathrm{r}$.

(Schluss; siehe Seite $81-90$ )

Ord. Stris ores.

F A M. TROCHILIDAE.

SUBF AM. POL YTMINAE.

Rhamphodon naevius (Dumont) Less. - Trochilus naevius Dumont, Euler No. 220.

- Glaucis hirsuta (Gm.) Gray. - Trochilus ferrugineus Pr. Max., Euler No. 174. ठ.

Pygmornis eremita (Gould), Cab. Hein. - Trochilus brasiliensis Pr. Max., Temm. nec Lath., Euler No. 165.

Phaëthornis squalida (Natt., Temm.) Jard. - Trochilus squalidus Temm., Euler No. 173.

Phaethornis eurynome (Less.) Jard. - Trochilus eurynomus Less., Euler No. 191.

\section{SUBFAM. LAMPORNITHINAE.}

+ Lampornis mango (Lin.) Sws. - Trochilus mango Lin., Euler No. 147. ठ. ․ ․ juv.

Thalurania glaucopis (Gm.) Bp. - Trochilus glaucopis Lin., Euler No. 27. ठ. ㅇ․

Petasophora serrirostris (Vieil.) Gray. - Trochilus crispus Spix., serrivostris Pr. Max., Euler No. 216. ठ.

SUBF AM. HELIOTHRICHINAE.

Heliothrix auriculata (Licht., Nordm.) Gray. - Trochilus auritus (nec Gm.), Euler No. 14, 221 ठ ad., ठ jun. juv.

SUBFAM. HYLOCHARINAE.

Agyrtria albiventris (Less.) Rchbch. - Euler ohne No. Agyrtria brevirostris (Less.) Rchbch. - Trochilus versicolor Licht. - Trochilus sp. Euler No. 76 ․

Hylocharis cyanea (Vieill.) Gray. - Trochilus sp. Euler No. 76 万.

Chlorostilbon nitidissimus (Licht.) Cab., Hein. Trochilus prasinus (nec Less.) Gould. - Ti. Pucherani Bourc. Tr. bicolor Gm.?, Euler No. 172. ठ.

Cab. Journ, f, Ornith, XXII. Jahrg. No. 126. A pril 1874. 


\section{SUBFAM. TROCHILINAE.}

Calliphlox amethystina (Gm.) Rchbch. - Troch. amethystinus Pr. Max., Euler No. 217. ठ.

SUBFAM. ORTHORHYNCHINAE.

Cephalolepis Delalandi (Vieil.) Rchbch. - Tr. Delalandi Vieil., Euler No. 218. ठ.

Lophornis magnifica, (Vieil.) Bp. - Tr. magnificus Vieil., Euler No. 77. ठ.

Prymnacantha Langsdorffi (Vieil.) Cab. Hein. Trochilus Langsdorffi Vieil., Euler No. 219. ठ.

F A M. CAPRIMULGIDAE.

$+\quad$ Nyctidromus albicollis (Gm.). - N. americanus Cass., nec Gm. - Caprimulgus guianensis Gm., Euler No. 23. ठิ.

Hydropsalis (Macropsalis) forcipatus (Nitzsch) Burm. - Caprimulgus sp. Euler No. 164. †. ठ juv.?

+ Nyctibius aethereus (Wied) Gray. - Caprimulgus aethereus Pr. Max., Euler No. 10. $\delta$.

Ord. Scansores.

F A M. $C U C U L I D A E$.

SUBFAM. COCCYGINAE.

+ Coccygus Euleri Cab., Journ. f. Orn. 1873, p. 72. - Coccygus seniculus (nec Vieil.) Euler No. 204.

Pyrrhococcyx macrurus (Gamb.) Cab. - Coccyzus cayauss (nec Lin.), Euler No. 13. ठ. ․

Neomorphus Geoffroyi (Temm.) Cab., Hein. - Coccyzus Geoffroyi Temm., Euler No. 44. ठ. ․․

Diplopterus naevius (Lin.) Gray. - Coccyzus naevius Temm., Euler No. 59. ठ.

Dromococcyx pavoninus Natt., Pelz. - Dromococcyx phasianellus (nec Spix) Burm., Euler No. 192. ‥

SUBFAM. CROTOPHAGINAE.

Octopteryx guira (Gm.) Cab., Hein. - Coccyzus Guira Temm., Euler No. 156. $\delta$.

Crotophaga ani Lin. - Euler No. 137. ठ.

F A M. $B U C C O N I D A E$.

SUBFAM. BUCCONINAE.

Nonnula rubecula (Spix) Sclat. - Bucco rubecula Spix, Euler No. 91.

Malacoptila torquata (Hahn) Sclat. - Capito fuscus (nec Gm.) Wied, Euler No. 24. ठ. + . 
Uebersicht der von C. Eu l er in Cantagallo gesammelten Vögel. 227

Notharchus Swainsoni (Gr.) Cab., Hein. - Capito macrorhynchus (nec Gm.), Euler No. 193. ठ.

F A M. TROGONI DAE.

-Pothinus atricollis (Vieil.) Cab., Hein. - Trogon atricollis Vieil., Euler No. 38. o. ㅇ.

Aganus aurantius (Spix) Cab, Hein. - Troyon ourucui (nec Lin.), Euler No. 108. ठ. +.

Aganus viriais (Lin.) Cab., Hein. - Trogon violaceus (nec Lin.) Spix, Wied, Euler No. 3. .. .

\section{F A M. $G A L B U L I D A E$.}

Cauax tridactylus (Pall.) Cab. - Galbula tridactyla Licht., Euler No. 94. .

F A M. P IC I D A E.

SUBFAM. PICUMNINAE.

Picumnus cirratus Temm. - P. minutissimus Wied, Euler No. 177. ठ.

\section{SUBFAM. DRYOCOPINAE.}

Ceophloeus lineatus (Lin.) Cab. - Picus lineatus Lin., Euler No. 206. $\delta$. .

Ceophloeus erythrops (Cuv.) Cab. - Euler No. 206. ठ. - Herr Euler hat diese seltnere Art, von der nur ein $\delta$ im Balge vorhanden war, von der vorhergehenden nicht unterschieden.

Phloeoceastes robustus (Freyr., Licht.) Cab. -- Picus robustus Licht., Euler No. 31. ठ. †.

\section{SUBFAM. CHRYSOPTILINAE.}

Campias maculifrons (Spix) Cab., Hein. - Picus affinis Sws. pt. Sundev. - P. passerinus (nec Lin.) Wied, Euler No. 4. $\delta$.

Craugasus erythropes (Vieil.) Cab., Hein. - Picus erythrops Vieil., Euler No. 155. ठ. ․

Craugasus polyzonus (Valenc.) Cab., Hein. - Picus sp. Euler No. 149.

Chrysoptilus cristatus (Vieil.) Cab., Hein. - Picus chlorozostus Wagl. - P. melanochlorus (nec Lin., Gm.,) Licht., Wied, Euler No. 19. $\delta$.

SUBFAM. GECININAE.

Celeus flavescens (Gm.). - Picus favescens Gm., Euler No. 293. ठ.

SUBFAM. MELANERPINAE:

Tripsurus flavifrons (Vieil.) - Picus coronatus Illig., Euler No. 47. $\delta$. ㅇ. 
Leuconerpes candidus (Otto) Sws. - Picus dominicanus Vieil., Licht. - P. candidus Otto, Euler No. 96. §. $q$.

F A M. RHAMPASTIDAE.

SUBFAM. RHAMPHASTINAE.

Selenidera maculirostris (Licht.). - Pteroglossus maculirostris Cuv., Temm., Euler No. 37. ठ. +.

Pteroglossus Wiedii Sturm. - Pteroglossus Arassari (nec Illig.) Euler No. 60. $\delta$.

Pteroglossus Baillonii Vieil., Temm., Wagl. - Euler No. 197. ठ. ‥

Rhamphastos dicolorus Lin. - Euler No. 71. ठ. ㅇ.

Rhamphastos Temminckii Wagl. - Euler No. 25. ठ. ㅇ. F A M. PSITTACIDAE.

Conurus propinquus Sclat., Cat. p. 346. _- Psittacus guianensis (nec Lin.) Wied, Euler No. 86. ठ. +.

Microsittace cruentata (Neuw.) - Psittacus cruentatus Pr. Max., Euler No. 232. ㅇ.

Microsittace vittata (Shaw). - Psittacus vittatus Shaw, Euler No. 198. $q$.

Brotogerys tiriacula (Bodd.) - Psittacus tirica Gm. Ps. viridissimus Kuhl, Temm., Euler No. 185. ठ.

Chrysotis farinosa (Bodd.). - Psittacus pulverulentus Gm., Euler No. 176. ㅇ.

Pionus Maximiliani (Kubl) Bp. - Psittacus flavirostris Spix, Euler No. 61. $q$.

Psittacula passerina (Lin.). - Psittacus passerinus Lin., Euler No. 57. §. + .

Ord. Raptatores.

F A M. STRIGIDAE.

4 Glaucidium ferrugineum (New.) Kaup., Cab., Journ. f. Orn. 1869, S.206. - Strix ferruginea Pr. Max., Euler No. 189. ठ.

F A M. FALCONIDAE.

SUBFAM. ACCIPITRINAE.

Nisus erythrocnemius (Gray). - Falco Beskei Licht. Falco sp. Euler No. 180. $q$ ad.

Hieraspizia tina (Lath.). - Falco tinus Lath. - Falco sp. Euler No. 188. §. No. 179. ․ - Iris kirschroth. Weibchen bedeutend grösser.

+ Dinospizias pectoralis. - Astur pectoralis (Cuv.) 
Uebersicht der von C. Eu ler in Cantagallo gesammelten Vögel. 229

Bp., v. Pelz. - Accipiter pectoralis Sclat. - Falco sp. Euler No. 163. đ. - Herrn Euler verdanken wir die erste specielle Angabe eines Fundortes dieser seltenen, erst in wenigen Exemplaren bekannten Art. Hierdurch wird Bonaparte's Angabe, dass der Vogel nicht aus Indien, sondern aus Brasilien stamme, bestätigt. Auffallend ist die von Bonaparte herrorgehobene Aehnlichkeit in der Färbung mit Spizaëtus ornatus. Der Vogel macht den Eindruck eines kühnen, starken Räubers, und scheint die hier vorgeschlagene eigene subgenerische Stellung zu beanspruchen. Er passt zu keiner amerikanischen Form der Accipitrinen.

Rupornis Nattereri (Sclat. et Salv.). - Falco magnirostris (nec Lin.), Euler No. 53. $\delta$. + .

- Climacocercus ruficollis (Vieil.). - Falco xanthothorax et leucauchen Temm. - Falco sp. Euler No. 26. o. q. Iris graubraun.

-Harpagus diodon (Temm.). - Falco diodon Temm., Euler No. 183. 万. ‥

SUBFAM. FALCONINAE.

-Tinnunculus (Poecilornis) gracilis (Sws.) Cab. Falco sparverius (nec Lin.), Euler No. 107. 8. क.

Hypotriorchis deiroleucus (Temm.). - Falco sp. Euler No. 213. $q$ ?.

SUBFAM. MILVINAE.

Ictinia plumbea (Gm.) Vieil. - Falco plumbeus Lin., Euler No. 102. t. $^{\text {? }}$.

Nauclerus furcatus (Lin.) Vig. - Falco Yetapa Pr. Max., Euler No. 124. ‥

Odontriorchis cayennensis (Gm.). - Falco palliatus Pr. Max., Euler No. 126. o.

SUBFAM. BUTEONINAE.

Buteola brachyura (Vieil.). - Buteo albifrons (Neuw.) Schleg. - Asturina albifrons Kaup. - Falco albifrons Pr. Max., Euler No. 205.

\section{SUBFAM. AQUILINAE.}

Spizaetus melanoleucus (Vieil.) Gray. - Falco atricapillus Cuv., Temm. - Falco sp. Euler No. 222. ․

Morphnus harpyia (Gm.) Cab. - Thrasaetus Harpyia Gray. - Falco destructor Daud., Euler No. 234. ㅇ. 


\section{SUBFAM. POLYBORINAE.}

- Hypomorphnus unicinctus (Temm.) Cab. - Faloo sp. Euler No. 127. $q$. juv.

Milvago chimachima (Vieil.). - Falco degener Illig., Euler No. 39. $\delta$ jun. $q$ juv.

\section{Ord. Gy ratores.}

F A M. C O L U M B ID A E.

Zenaida maculata (Vieil.) Gr. - Columba sp. Euler No. 103. ㅇ.

+ Peristera cinerea (Temm.) Sws. - Columba cinerea Temm., Euler No. 215. ठ.

Peristera Geoffroyi (Temm.) Gr. - Columba Geoffroyi Temm., Euler No. 30. ठ. ․

Leptoptila rufaxilla (Richard) Bp.? - Peristera frontalis (Temm.) Burm. - Peristera macrodactylus Gray. - Columba rufaxilla Wagl., Euler No. 92. ð.

- Oreopelia violacea (Temm.). -. Columba montana Auct., Euler No. 109. ठ.

Oreopelia montana (Lin.) Bp. - Columba montana Euler No. 109. ð. - Herr Euler hat die beiden vorstehenden Arten nicht unterschieden.

Ord. Ras ores.

F A M. TETRAONIDAE.

SUBFAM. ODONTOPHORINAE.

Odontophorus dentatus (Temm.). - Perdix dentata Temm., Euler No. 95. ठ.

$$
\text { F A M. } C R A C I D A E \text {. }
$$

Penelope (Salpiza) jacquaçu Spix. - Penelope cristata Lath., Wagl., nec Lin., Euler No. 100. đ.

\section{Ord. Cursores.}

\section{F A M. CRYPTURIDAE.}

Crypturus noctivagus (Neuw.) Wagl. - Tinamus noctivagus Pr. Max., Euler No. 74. ठ.

Crypturus pileatus (Bodd.). - Crypturus Sovi (Gm.) Licht. - Tinamus Sovi Lath., Euler No. 101. ठ.

Crypturus Tataupa Illig. - Tinamus Tataupa Temm., Euler No. 178. $\delta$.

Crypturus obsoletus (Temm.) Licht. - Tinamus obsoletus Temm., Euler No. 202. 


\section{Ord. Grallatores.}

-Vanellus cayanensis (Gm.) Gray. - Euler No. 214.

Parra jaçana Lin. - Euler No. 148. đo jun. juv.

Porzana melanophaea (Vieil.) Sclat., Salv. - Crex lateralis Lieht. - Gallinula lateralis Pr. Max., Euler No. 236.

+ Porzana albicollis (Vieil.) Sclat., Salv. - Rallus albicollis Vieil. - Crex mustelina Licht. - Ortygometra albicollis (Vieil.) Euler No. 106.

Ortygarchus plumbeus (Vieil.) - Gallinula Sarracura Spix. - Crex melanura Licht. - Aramides plumbeus. Burm. Gallinula plumbea Vieil., Euler No. 34. ․

+ Forphyrio martinica (Lin.). - Gallinula martinica Lath. - Gallinula martinicensis Pr. Max., Euler No. 105, ad. juv.

Ord. Natatores.

F A M. $A N A T I D A E$.

Querquedula brasiliensis (Gm.) Cab. - Anas brasiliensis Lin., Euler No. 75. ㅇ.

Die Zahl der von Herrn Euler im District Cantagallo gesammelten Arten beläuft sich nach vorstehender Uebersicht auf 228.

$\mathrm{Nachschrift.}$

Unsere weiter vorn, Seite 84 Note, ausgesprochene Vermuthung, dass das ausgefärbte Männchen der Sporophila falcirostris grau sein würde, hat sich bestätigt. Herr von Pelzeln hat soeben das bisher unbekannte alte Männchen dieser Art beschrieben, wie folgt:

„Spermophila falcirostris (Temm.) ठ: Plumbeus pileo obscuriore, speculo alari albo, gula, tectricibus alarum inferioribus posterioribus et abdomine medio albis, tectricibus caudae inferioribus flavido-albis, rostro (in specimine exsiccato) flavo, pedibus corneis. Longit. 4", longit. culminis rostri a fronte secundum curvaturam $4^{1 / 3} 3^{\prime \prime \prime}$, rostri a rictu linea recta $5^{\prime \prime \prime}$, altitudo maxillae $1{ }^{1 / 2}{ }^{\prime \prime \prime}$, mandibulae $2{ }^{1} 3^{\prime \prime \prime}$, longit. alae $2^{\prime \prime} 5^{\prime \prime \prime}$, cauda $1^{\prime \prime} 10^{\prime \prime \prime}$. Hab.: Bahia et Nov. Friburgia." v. Pelzeln, Separat-Abdr. aus „Nunquam otiosus", p. 291-292. 


\section{$2 \mathrm{BHL}$ Biodiversity Heritage Library}

1874. "Uebersicht der von Herrn Carl Euler im District Cantagallo, Provinz Rio de Janeiro, gesammelten Vögel." Journal $f u$

r Ornithologie 22, 225-231. https://doi.org/10.1007/bf02007184.

View This Item Online: https://www.biodiversitylibrary.org/item/101706

DOI: https://doi.org/10.1007/bf02007184

Permalink: https://www.biodiversitylibrary.org/partpdf/141321

\section{Holding Institution}

Smithsonian Libraries

\section{Sponsored by}

Biodiversity Heritage Library

\section{Copyright \& Reuse}

Copyright Status: Public domain. The BHL considers that this work is no longer under copyright protection.

This document was created from content at the Biodiversity Heritage Library, the world's largest open access digital library for biodiversity literature and archives. Visit BHL at https://www.biodiversitylibrary.org. 